\title{
Image Deconvolution Ringing Artifact Detection and Removal via PSF Frequency Analysis
}

\author{
Ali Mosleh ${ }^{1}$, J.M. Pierre Langlois ${ }^{1}$, and Paul Green ${ }^{2}$ \\ 1 École Polytechnique de Montréal, Canada \\ 2 Algolux, Canada \\ \{ali.mosleh,pierre.langlois\}@polymtl.ca, paul.green@algolux.com
}

\begin{abstract}
We present a new method to detect and remove ringing artifacts produced by the deconvolution process in image deblurring techniques. The method takes into account non-invertible frequency components of the blur kernel used in the deconvolution. Efficient Gabor wavelets are produced for each non-invertible frequency and applied on the deblurred image to generate a set of filter responses that reveal existing ringing artifacts. The set of Gabor filters is then employed in a regularization scheme to remove the corresponding artifacts from the deblurred image. The regularization scheme minimizes the responses of the reconstructed image to these Gabor filters through an alternating algorithm in order to suppress the artifacts. As a result of these steps we are able to significantly enhance the quality of the deblurred images produced by deconvolution algorithms. Our numerical evaluations using a ringing artifact metric indicate the effectiveness of the proposed deringing method.
\end{abstract}

Keywords: deconvolution, image deblurring, point spread function, ringing artifacts, zero-magnitude frequency.

\section{Introduction}

Despite considerable advancements in camera lens stabilizers and shake reduction hardware, blurry images are still often generated due to the camera motion during the exposure time. Hence, effective restoration is required to deblur captured images. Assuming that the imaging system is shift invariant, it can be modeled as

$$
b=l \oplus k+\omega,
$$

where $b \in \mathbb{R}^{M N}$ is the blurred captured image, $l \in \mathbb{R}^{M N}$ is the latent sharp image, $k \in \mathbb{R}^{M N \times M N}$ is the point spread function (PSF) that describes the degree of blurring of the point object captured by the camera, $\omega \in \mathbb{R}^{M N}$ is the additive noise, and $\oplus$ denotes the $2 \mathrm{D}$ convolution operator. Hence, the objective of the deblurring process is to recover $l$ from $b$. Image deconvolution is often used for that purpose. One may neglect the noise and consider a naive solution for this inverse problem as

$$
l=\mathcal{F}^{-}\left(\frac{\mathcal{F}(b)}{\mathcal{F}(k)}\right)
$$

D. Fleet et al. (Eds.): ECCV 2014, Part IV, LNCS 8692, pp. 247-262, 2014.

(C) Springer International Publishing Switzerland 2014 

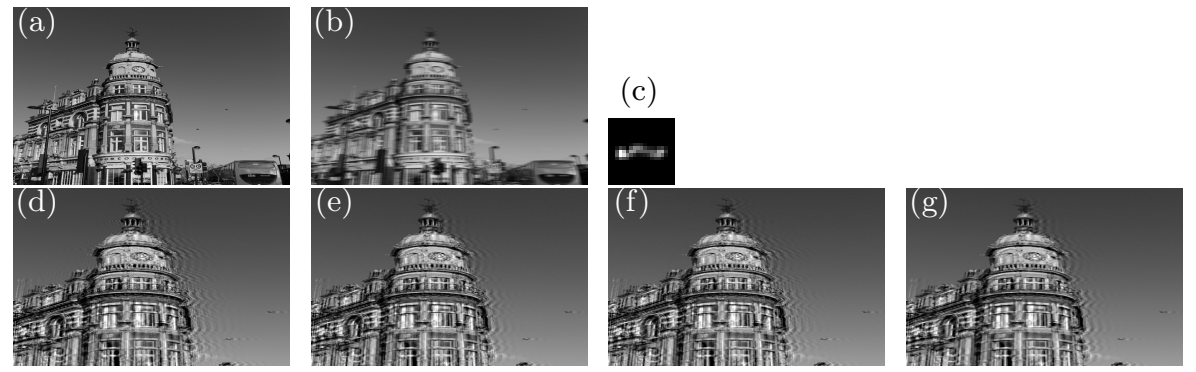

Fig. 1. Deblurred images with ringing artifacts. (a) Ground-truth image. (b) Degraded image. (c) Blur kernel (PSF). (d)-(g) Deblurring results using RL, [6], [12], and [21]. The images are rescaled for a better illustration. (The figures in this paper are best viewed on screen, rather than in print).

where $\mathcal{F}($.$) denotes the 2D Fourier transform operator. Even if the PSF k$ is known precisely, this approach results in amplified singularities in the presence of a very small amount of noise. This occurs due to the characteristics of blur kernels and the unknown Fourier transform of the random noise function [1]. Hence, numerous deconvolution algorithms have been developed to estimate the latent sharp image. They include Wiener [2] and Richardson-Lucy (RL) [3, 44] techniques, least squares minimization [5], Bayesian inference [6 8], advanced variational based [9 13], and stochastic framework [14] methods.

A challenging problem in latent image restoration is the presence of wavelike artifacts called ringing that appear near strong edges. Ringing examples are illustrated in Fig. 1. These light and dark ripples are mainly due to the Gibbs phenomenon [15] as the Fourier sums overshoot at discontinuities (i.e., image edges), and this overshoot does not die out while the frequency increases. This phenomenon can be amplified if the noise $\omega$ is not modeled accurately and if the PSF $k$ is noisy or inaccurately estimated 6]. The latter case is even more serious in blind deconvolution approaches [16 19] that involve PSF estimation from the blurred image.

Several researchers have considered the ringing issue and tried to reduce the artifacts in deconvolution schemes. Yuan et al. 20] addressed ringing artifacts by adapting edge-preserving bilateral filters to the conventional RL algorithm in a residual multiscale deconvolution approach. Shan et al. [6] proposed a spatially random noise model to separate errors in noise and PSF estimation. They also proposed to impose a smoothness constraint on the latent image to suppress ringing artifacts in the deconvolution process. Cho et al. [21] recognized saturated pixels, pixels degraded by non-Gaussian noise, and a non-linear camera response function violate the linear blur model of Eq. (1) and cause severe ringing artifacts. The camera temporal shutter modulation proposed by Raskar et al. 22] generates a very flat frequency spectrum. This eliminates zeros from the PSF to overcome the occurrence of zeros in the denominator in the deconvolution process and consequently to reduce artifacts. The idea of taking into 
account the zero frequencies of the PSF was also employed in the design of the coded aperture of Levin et al. 23] in removal of the out of focus blur. Increasing the weight of the regularizer term in the variational-based deconvolution techniques can also suppress the ringing artifacts. However, this over-smooths the recovered image and removes its edges 10 12]. Employing appropriate priors in such regularization schemes may reduce the potential artifacts as done partially by using cross-channel priors introduced by Heide et al. 24]. A means of ringing artifact detection can be quite effective to add ringing a priori knowledge to the deconvolution algorithm.

Ringing artifact detection algorithms were initially developed to evaluate the quality of compressed images impaired by ringing artifacts [25, 26]. These algorithms are not practical in detection of ringing artifacts produced in the deconvolution process, since the nature of ringing artifacts is different in image compression and image deconvolution. This yields a need for deconvolutionspecific algorithms to detect ringing artifacts. The deblurred image is passed through a bank of Gabor-like wavelets by Zuo et al. [27], then the vertical and horizontal oscillation peaks are counted in the image filter responses as a metric for ringing artifacts. Since the employed filters are neither adapted to the content of the image nor to the PSF, high frequency patterns and textures in the image are falsely classified as artifacts in the filter response. A ringing detection procedure was proposed by Liu et al. 28. It consists of generating a pyramid of different scales of the recovered image and finding the gradient difference between each level of the pyramid. Such ringing artifact detectors are appropriate only for quality assessment of the deblurred image and are not directly involved in an approach to produce artifact-free deblurred images.

In this paper, we first propose a deconvolution ringing artifact detection scheme based on the inspection of the saddle points and identifying the zeros in the PSF frequency response. Specific 2D Gabor wavelets with appropriate directionality and wavelength properties are then generated for the PSF components responsible for ringing artifacts. The produced $2 \mathrm{D}$ wavelets are used to localize the artifacts in the deblurred image. Next, the ringing artifacts are removed from the image by employing the produced wavelets in a variational-like regularization algorithm. We in fact suppress the artifacts by minimizing the image response to the generated Gabor filters. We introduce a priori knowledge of the ringing artifact locations in the restored image, by taking into account frequency details of the PSF and employing Gabor filters. The deblurring process independence of the proposed method is so that it can be applied on the result of any deconvolution approach. We show that besides its simplicity, this scheme significantly enhances the deconvolution results in terms of ringing artifact reduction which is still a challenge in image restoration.

The rest of the paper is organized as follows. In Section 2, the PSF frequency analysis and ringing artifact detection scheme is presented. In Section 3, a regularization algorithm is introduced to remove the artifacts by employing the artifact detection filters in a variational-based minimization. We then present experimental results in Section 4, followed by our conclusions in Section 5. 

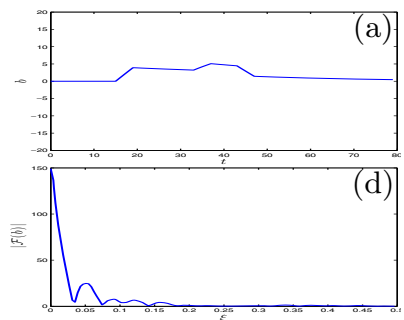

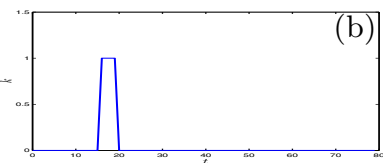

(e)

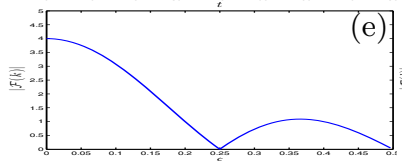

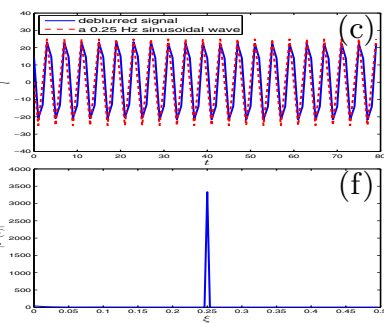

Fig. 2. 1D signal restoration using Eq. (2) (a-c) Blurred signal, PSF, and deblurred signal in the time domain. (d-f) Blurred signal, PSF, and deblurred signal in the frequency domain.

\section{Ringing Artifact Detection}

The principle of deblurring consists of decoupling the blur function from the observed image as in Eq. (2). However, mainly since the noise is neglected, this model generates severe artifacts. Although this model has been modified to include reasonable noise models in the modern deconvolution techniques, the inevitable presence of zero values in the denominator of Eq. (2) is a problematic source of ringing artifacts. This issue is shown in Fig. 2 for a 1D signal restoration. A 1D signal is convolved with the PSF shown in Fig. 2(b) to generate the blurred signal Fig. 2(a). This signal is then deblurred using Eq. (2) and the result is shown in Fig. 2(c). By inspecting the Fourier transform of the PSF (Fig. 2(e)) we find that at the frequency of $0.25 \mathrm{~Hz}$ the PSF has zero magnitude. In other words, PSF components that belong to the $0.25 \mathrm{~Hz}$ band have very low power. These components generate large amplitude oscillations at the same frequency in the deblurred signal (Fig. 2(f)). Therefore, in the time domain of the deblurred signal, components that belong to the $0.25 \mathrm{~Hz}$ frequency band dominate the signal. This effect appears as a sinusoidal pattern with a high amplitude in the deblurred signal and is considered a ringing artifact. From the large amplitude of the reconstructed signal Fig. 2(c) (-20 to 20) compared to that of the blurred signal Fig. 2(a) (0 to 5) we can infer how corrupted the deblurring result is. Such zero power frequency components in a 2D PSF can be largely responsible for disturbing ringing artifacts in $2 \mathrm{D}$ signals i.e., images. The fact that ringing artifacts follow a sinusoidal pattern inspired the deconvolution artifact detection techniques of Zuo et. al [27] and Liu et al. [28]. This is even more promising that from our PSF frequency analysis we can predict the frequency of this sinusoidal wave. This is shown for the 1D case in Fig. 2(c) by a superimposed sinusoidal signal indicated by a red diagram whose frequency of $0.25 \mathrm{~Hz}$ is chosen by inspection of the PSF frequency spectrum Fig. 2 (e).

\subsection{Using Gabor Filters}

The Gabor filter is a traditional choice for obtaining localized frequency information. A 2D Gabor filter offers an efficient localization of spatial and frequency 
information. Frequency and orientation representations of 2D Gabor filters have been shown to be appropriate for texture discrimination [29]. Since the impulse response of a Gabor filter is a sinusoidal wave plane, it can be an effective tool to detect the image ringing artifacts that follow sinusoidal patterns. The 2D Gabor filter is defined as

$$
g(x, y)=\frac{1}{2 \pi \sigma_{x} \sigma_{y}} e^{-\frac{1}{2}\left(\frac{x^{2}}{\sigma_{x}^{2}}+\frac{y^{2}}{\sigma_{y}^{2}}\right)} e^{-j 2 \pi\left(\left(u_{0} x+v_{0} y\right)+\phi\right)} .
$$

The Gabor function can be viewed as a sinusoidal plane of particular frequency and orientation, modulated by a Gaussian envelope. In Eq. (3) $(x, y)$ denotes the spatial location, $\sigma_{x}$ and $\sigma_{y}$ are respectively the horizontal and vertical standard deviations of the Gaussian envelope, $u_{0}$ and $v_{0}$ are respectively the x-axis and $y$-axis frequencies of the sinusoidal plane, and $\phi$ is the phase offset.

The spatial frequency of the ringing artifacts in a deblurred image can be determined by inspecting the Fourier transform of the PSF. The coordinate of a zero value in the Fourier transform of the PSF represents the spatial frequency of the ringing components in the deblurred image. Substituting $u_{0}$ and $v_{0}$ in Eq. (3) by the spatial frequency of a zero magnitude frequency component of the PSF results in an appropriate filter to localize the image artifacts produced by that frequency component. The parameters $\sigma_{x}$ and $\sigma_{y}$ of the Gaussian kernel in the filter can be selected based on the deblurred image contents. They should not be either too high to taper the image edges and misclassify them as ringing artifacts, or too low to miss the low intensity ringing artifacts. We need to ensure that the image ringing pattern with a delay or an advance (different phase offsets) will be detected by the filter. Hence, first one filter is produced with $\phi=0$ and another one is generated with $\phi=\pi / 2$, then the generated filters with different phase offsets are superimposed to make a single filter.

As an example of the process consider Fig. 3. where a blurred synthetic image and its PSF are shown in Fig. 3(b). This image is deblurred using the RL method as illustrated in Fig. 3(c). As seen in this figure, compared with the ground-truth image Fig. 3(a), the deblurring result contains ringing artifacts especially in the brighter region of the image. In order to have a better insight, the diagram of the first 140 pixel intensities at row 200 of the deblurred image is shown by the blue plot in Fig. 3(g). From the frequency spectrum of the PSF Fig. 31(e), the lowmagnitude component with spatial frequencies $u_{0}=-0.045$ and $v_{0}=0.001$ is picked as the source of ringing artifacts. (The procedure to select such frequencies is discussed in Section 2.2.) The Gabor filter exhibited in Fig. 3(d) is generated using Eq. (3) by utilizing these horizontal and vertical frequencies. The filter is then convolved with the deblurred image to localize the sinusoidal artifacts. The thresholded filter response of the deblurred image Fig. $3(\mathrm{~h})$ reveals the regions that contain artifacts in the deblurred image. The Gabor filter response at row 200 of the image is illustrated in Fig. 3(g) (red dashed plot), along with the deblurred pixel intensities (blue plot). In this case, the Gabor filter generated with the frequencies obtained from the PSF, effectively fits and localizes the wave-like artifacts produced in the deconvolution process. 

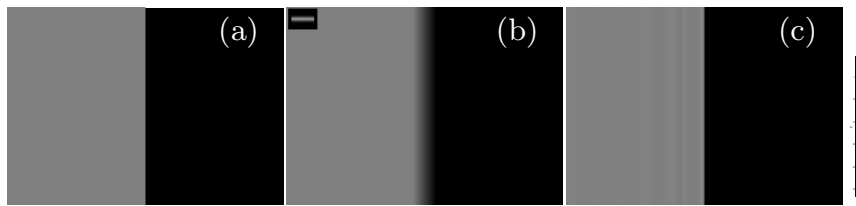

(d)
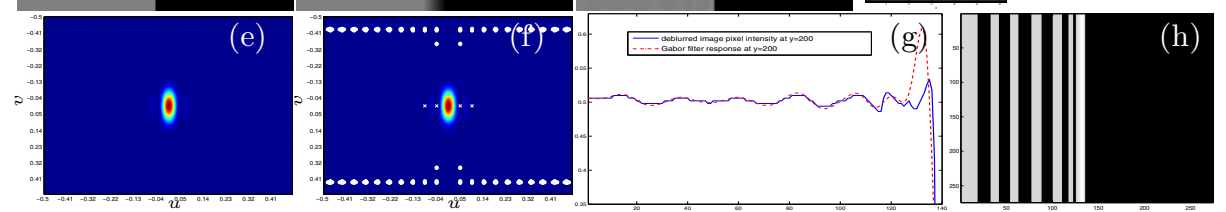

Fig. 3. Ringing detection by employing Gabor filter and the PSF frequency details. (a) Ground-truth image (originally $256 \times 256$ ). (b) Synthetically blurred image and its PSF. (c) Deblurred image that contains ringing artifacts. (d) Generated Gabor filter for artifact detection (enlarged for a better illustration). (e) The blur PSF in Frequency domain. (f) Saddle points and zero points in the frequency spectrum of the PSF. (g) Pixel intensity values and Gabor filter response values on row 200 of the image. (h) Thresholded image Gabor filter response to locate the ringing regions

\subsection{Artifact Detection Algorithm}

The ringing artifact detection algorithm first analyses the PSF to locate the zero-magnitude components in the Fourier domain. Then, for each component a Gabor filter is generated. There may exist many zero-magnitude frequency components in the PSF but not all of them are useful in the artifact detection process. The zero values located far from the center of the frequency spectrum may cause ringing but the frequency of their produced ringing is too high to be perceivable. Therefore, we avoid them in the process to reduce the number of Gabor filters employed in the scheme. Such kind of frequency components are marked with the white dots in the Fourier transform of the PSF shown in Fig. 3(f). Also, a sharp transient in the frequency domain of the PSF denotes an apparent oscillatory decay in the PSF which is the cause of the dominant ringing in the restored image. Hence, we reduce the number of required Gabor filters to the number of zero-magnitude frequency components of the PSF that lie on the saddle points of the PSF Fourier transform. This is done by creating a map of local minimum points in the Fourier domain using a morphological operator:

$$
m\left(\dot{p}, q^{\prime}\right)=\left\{\begin{aligned}
& 1 \quad \text { if }\left(\hat{K}\left(\hat{p}^{\prime}, q^{\prime}\right)<\hat{K}(p, q), \forall p \in[p-\ell, p+\ell] \wedge \forall q \in[q-\ell, q+\ell]\right) \\
& \wedge\left((p, q) \neq(\not{p}, \hat{q}) \wedge \hat{K}\left(\dot{p}, \hat{q}^{\prime}\right)<\delta\right) \\
& 0 \quad \text { otherwise },
\end{aligned}\right.
$$

where $\hat{K}=|\mathcal{F}(k)|$ denotes the magnitude matrix of the Fourier transform of the PSF indexed by $q$ and $p$, and $\ell$ determines the number of neighbouring values in $\hat{K} . \delta$ is a small value near zero used as a threshold to determine the low values, and $m$ is the map of local minima of $\hat{K}$. The white crosses in Fig. 3(f) are the appropriate points found by Eq. (4) in the PSF frequency spectrum. 


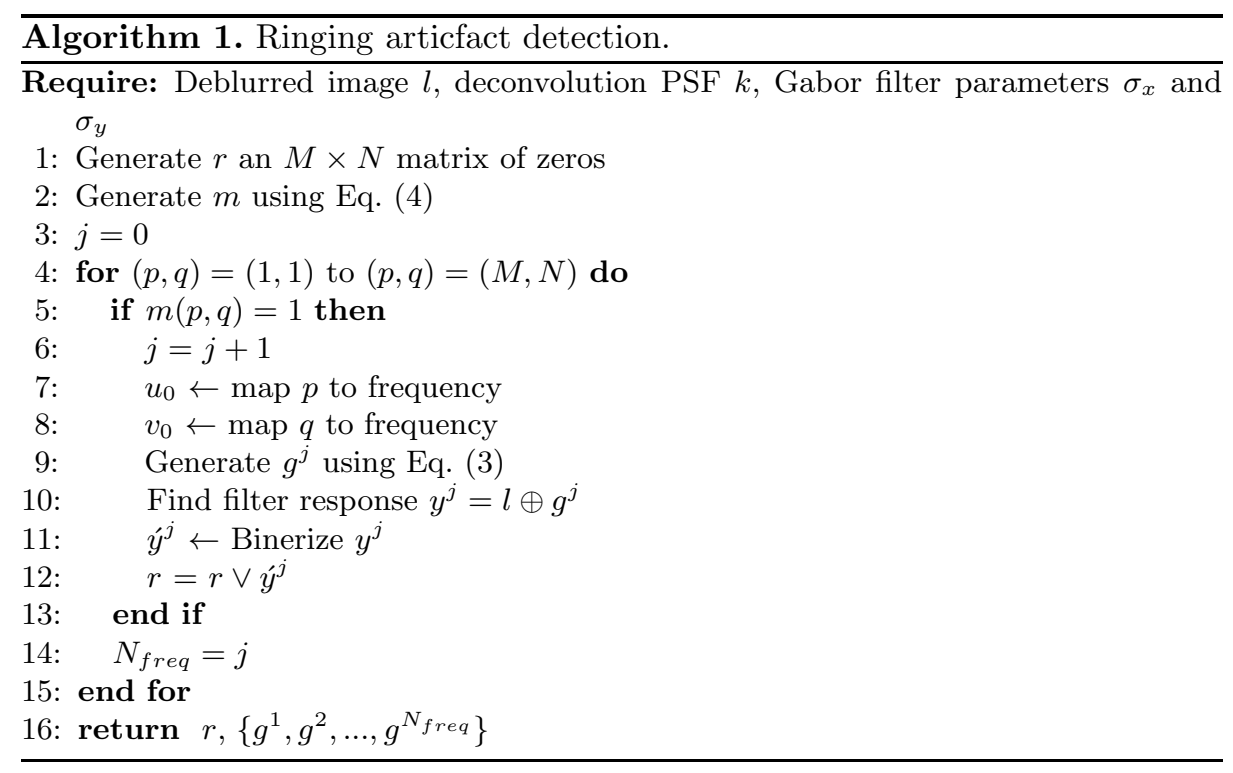

In the next step, a set of Gabor filters is generated using the spatial frequencies determined by $m$. Each filter is then applied on the deblurred image $l$ to produce different responses that represent ringing patterns with different frequencies and orientations. The filtering results are then binarized and superimposed to make a mask of all regions that contain ringing in the image. Algorithm 1 outlines all the steps of the ringing artifact detection method, where $\vee$ denotes the elementwise binary or operator to superimpose results. The principal objective of this algorithm is localizing the artifact regions. However, as will be discussed in the next section, in order to remove the artifacts we need the set of Gabor filters generated by Algorithm 1. Hence, in addition to the map of ringing regions $r$, the set of Gabor filters is returned by this algorithm for a later artifact removal procedure.

The ringing detection algorithm may falsely classify the image edges as part of the ringing pattern especially if the ringing occurs near strong edges. To avoid this issue, the process should be performed only on the smooth regions and not the edges. This cannot be done by simply running an edge detector to preserve the edges before the artifact detection step since the ringing artifacts would be detected by the edge detection schemes. Instead, we propose to analyse the local contrast of the image and run the algorithm on low contrast regions where there is no edge pixel. Typically, contrast is estimated by Weber's formula: $C_{l}=$ $\left(l_{o}-l_{b}\right) / l_{b}$ where $l_{o}$ and $l_{b}$ are the luminance of the object and its surrounding background, respectively. More complex contrast analysis can be performed by employing discrete cosine transform and wavelets [30, 31]. We use the local mean mean $(l)$ and standard deviation $s t d(l)$ of the image intensity to estimate the contrast of a region with its background [32] $C_{l}=\operatorname{std}(l) /$ mean $(l)$. This value is computed for all existing blocks (e.g., $4 \times 4$ pixels) in the input image, then the 
regions with large $C_{l}$ are removed from the binary mask generated at line 11 in Algorithm 1. Hence, edges are not detected as part of the ringing artifacts.

\section{$3 \quad$ Ringing Artifact Removal}

Let $g^{j}$ be a Gabor filter generated from the $j$ th zero-magnitude frequency component of the PSF. Hence, for a PSF employed in the deblurring process with $N_{\text {freq }}$ zero-magnitude frequency components, we generate a set of Gabor filters $g^{1}, g^{2}, \ldots, g^{N_{\text {freq }}}$ to detect the ringing artifacts in the deblurred image $l$. The ringing artifacts detected by analysing the PSF frequency components and Gabor filters can be suppressed through a regularization scheme. Our artifact suppression technique, similar to the total variation [9] and Tikhonov regularization [33] methods widely used in inverse imaging problems, consists of a likelihood term and a regularizer as follows:

$$
\underset{f}{\operatorname{minimize}} \frac{\mu}{2}\|f-l\|^{2}+\sum_{j=1}^{N_{\text {freq }}}\left\|g^{j} f\right\|_{1},
$$

where the left term is the likelihood function weighted by the regularization parameter $\mu$ and the right term is the regularizer. In (5) $l$ is the observed (already deblurred with ringing artifacts) image and $f$ is the reconstructed image. In fact, some a priori knowledge about the unknown image (i.e., ringing artifacts and their locations) is added to the likelihood function by employing the regularizer. Note that $\|.\|_{1}$ is the $L_{1}$ norm, $\|$.$\| is the L_{2}$ norm and $\|.\|^{2}$ is its square, and for brevity $g^{j} f \equiv f \oplus g^{j}$ where $\oplus$ denotes the $2 \mathrm{D}$ convolution operator.

Despite its simple formulation, problem (5) is computationally challenging to solve. This is mainly due to the non-differentiability and non-linearity of the regularizer [34]. According to [35] and [36], such regularization problems can be addressed using the half-quadratic penalty method. Therefore, we follow the variable splitting scheme employed in [10 12, 37] to solve (5). We introduce auxiliary variables $u=\left\{u^{1}, \ldots, u^{N_{\text {freq }}}\right\}$ to transfer $g^{j} f$ out of the non-differentiable term $\|\cdot\|_{1}$ in (5) to model the optimization as

$$
\underset{f, u}{\operatorname{minimize}} \frac{\mu}{2}\|f-l\|^{2}+\frac{\beta}{2} \sum_{j=1}^{N_{\text {freq }}}\left\|g^{j} f-u^{j}\right\|^{2}+\sum_{j=1}^{N_{\text {freq }}}\left\|u^{j}\right\|_{1},
$$

with a penalty parameter $\beta$. The value of this penalty parameter has an important role in convergence of (6) [10]. When $\beta \rightarrow \infty$, the solution of the approximation problem (6) converges to that of (5). Using the information $r$ obtained about the locations of the ringing regions, $\beta$ is adapted so that the regulirizer term has the least effect on the pixels that do not belong to the ringing regions. Consider $\beta$ as an $N \times M$ matrix whose elements indexed by $(x, y)$ defined as

$$
\beta(x, y)= \begin{cases}\beta_{0} & \text { if } r(x, y)=0 \\ \tilde{\beta} & \text { otherwise }\end{cases}
$$


where $\tilde{\beta} \rightarrow \infty$ and $\beta_{0}$ is a small constant in the deringing process. Hence, the weight of artifact-free pixels remains small in (6) and the ringing minimization is carried out only on the pixels identified by $r$ in Algorithm 1 Note that in (6) the multiplication is performed element-wise on $\beta$.

The optimization problem (6) can be performed by alternating between two steps in an iterative scheme. In one step we solve for $f$, given values of $u$ :

$$
\underset{f}{\operatorname{minimize}} \frac{\mu}{2}\|f-l\|^{2}+\frac{\beta}{2} \sum_{j=1}^{N_{\text {freq }}}\left\|g^{j} f-u^{j}\right\|^{2},
$$

and in another step we solve for $u$, given values of $f$ :

$$
\underset{u}{\operatorname{minimize}} \frac{\beta}{2} \sum_{j=1}^{N_{\text {freq }}}\left\|g^{j} f-u^{j}\right\|^{2}+\sum_{j=1}^{N_{\text {freq }}}\left\|u^{j}\right\|_{1} .
$$

which is in fact solving $N_{\text {freq }}$ sub-problems of:

$$
\underset{u^{j}}{\operatorname{minimize}} \frac{\beta}{2}\left\|g^{j} f-u^{j}\right\|^{2}+\left\|u^{j}\right\|_{1}
$$

\section{1 f Sub-problem}

Given $u$ which is obtained from the previous iteration, we need to solve (8) to approximate a new $f$. A fixed value for $u$ yields a quadratic form for (8). Hence, the solution for this problem can be the solution for the normal equation as

$$
\left(\sum_{j=1}^{N_{\text {freq }}} g^{j^{T}} g^{j}+\frac{\mu}{\beta} h\right) f=\left(\sum_{j=1}^{N_{\text {freq }}} g^{j^{T}} u^{j}\right)+\frac{\mu}{\beta} l
$$

where $h$ is an $N \times M$ matrix of ones. Using the Fourier transform, (11) can be rewritten as

$$
f=\mathcal{F}^{-1}\left(\frac{\sum_{j=1}^{N_{\text {freq }}} \mathcal{F}\left(g^{j}\right)^{*} \mathcal{F}\left(u^{j}\right)+\frac{\mu}{\beta} \mathcal{F}(l)}{\sum_{j=1}^{N_{\text {freq }}} \mathcal{F}\left(g^{j}\right) * \mathcal{F}\left(g^{j}\right)+\frac{\mu}{\beta} \mathcal{F}(h)}\right),
$$

where the multiplications are element-wise and $*$ denotes the complex conjugate.

\section{$3.2 u$ Sub-problem}

Finding an optimal $u$ is subject to minimizing (10) independently for each Gabor filter, in total $N_{\text {freq }}$ times. This minimization can be done using the conventional approaches such as Newton-Raphson. It was shown in [10, 37] that such type of problems can be reduced to a shrinkage scheme so we can avoid the computational challenges of the conventional optimization methods. Hence, each component of $u^{j}$ indexed by $i$ can be approximated as follows:

$$
u_{i}^{j}=\max \left(\left|\left(g^{j} f\right)_{i}\right|-\frac{1}{\beta_{i}}, 0\right) \operatorname{sgn}\left(g^{j} f\right)_{i},
$$

where $\max ($.$) returns the maximum and \operatorname{sgn}($.$) is the sign function.$ 

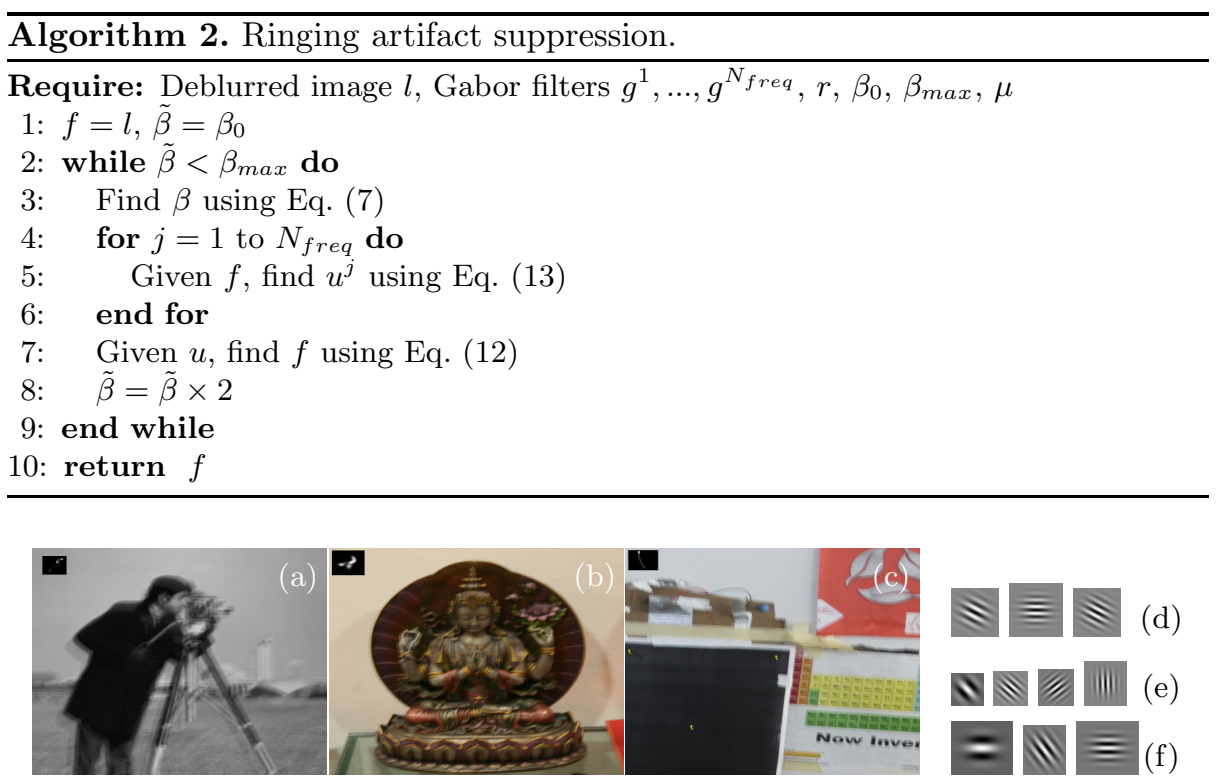

Fig. 4. Sample blurry images and their blur kernels used in our tests and the Gabor filters generated for artifact removal. (a) Image 1: The original image is synthetically blurred using the motion blur PSF available in 28]. (b) Image 2: The blurry image and its estimated PSF are provided by Shan et al. [6]. (c) Image 3: The blurry image and the PSF are captured using a dual-camera set-up. The kernels in (a), (b) and (c) are 4 times larger than the original sizes, for a better view. (d-f) Gabor filters used in artifact detection and removal for the image shown in (a), (b), and (c) respectively.

\subsection{Summary of the Artifact Removal Algorithm}

The optimization process is outlined in Algorithm 2, We minimize (6) by alternating between the $f$ and $u$ sub-problems. As mentioned before, for the optimization problem to converge we need to satisfy $\beta \rightarrow \infty$ for the corresponding ringing pixels. Therefore, we initiate $\tilde{\beta}$ with $\beta_{0}$ and increment it during the iterations. The iterations stop once $\tilde{\beta}=\beta_{\max }$. It is worth noting that the fixed parameters in Eq. (8) , such as $\mathcal{F}\left(g^{1}\right), \mathcal{F}\left(g^{2}\right), \ldots, \mathcal{F}\left(g^{N_{\text {freq }}}\right)$ and their conjugates, $\mathcal{F}(l)$, and $\mathcal{F}(h)$ can be precomputed to save some computational time.

\section{Experimental Results}

We evaluated the performance of the proposed artifact detection and removal scheme on images. To this end, a set of blurry images were used. Three different types of blurry image and PSF pairs belong to this set. The first type of blurry images were created synthetically with known PSFs, as presented in [28]. An example of such images and PSFs is shown in Fig. 4(a). Another type of images were captured by camera that contain motion (handshake) blur. Using a blind 

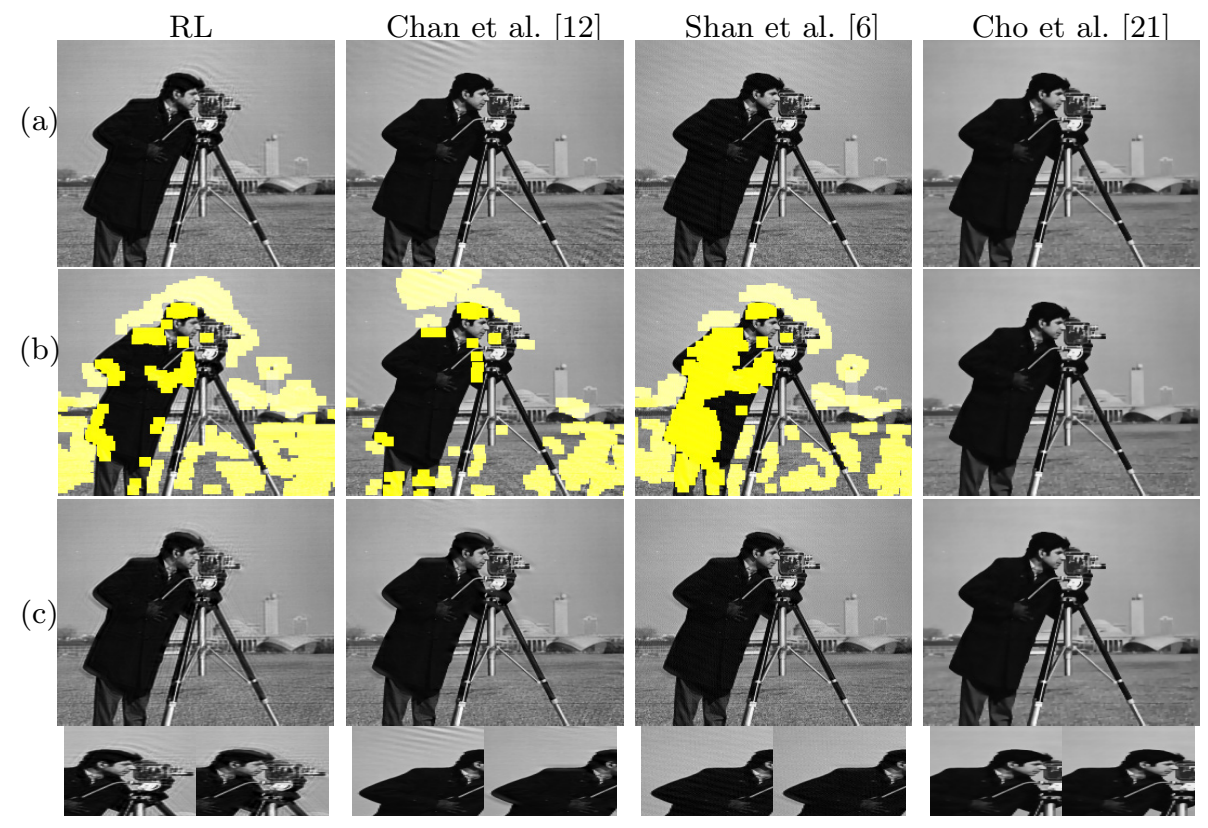

Fig. 5. Deblurring Image 1 synthetically blurred using a known PSF. (a) Deblurring results using different algorithms. (b) Detected ringing regions. (c) Deringing results.

deconvolution approach, a PSF was estimated for each image. An example of such images and their PSFs is shown in Fig. 4(b) provided by Shan et al. [6]. Finally, we built a dual-camera framework similar to that of Ben-Ezra and Nayar [38] to estimate the PSF from the camera motion in the exposure time. Fig. 4(c) shows an image and its corresponding PSF captured using this framework. The blurry images were deblurred by employing different algorithms: (i) RL [3, 4], (ii) Chan et al.'s method [12] that uses total variation norm prior and $L_{2}$ variant prior, (iii) the algorithm of Shan et al. [6] using a smoothness prior, and (iv) that of Cho et al. 21] by handling ringing prone pixels. The deblurring results are demonstrated in Fig. 5(a), Fig. 6(a) and Fig. 7(a). The input parameters for the deblurring algorithms [6, 12, 21] such as regularization weights and smoothness factors are chosen so that they do not generate over-smoothened and cartoonlike results. As seen in these figures, all of the employed deconvolution schemes generate ringing artifacts, except Cho et al.'s algorithm for Image 1. This image is synthetically blurred and its PSF is precise. Hence, it is deblurred well using Cho et al.'s method where ringing prone outliers are well-handled. Algorithm 1 was performed on the deblurred images to locate the artifacts generated in the deconvolution stage. Then, the filters produced by Algorithm 1 were used in Algorithm 2 to suppress the ringing artifacts.

In the ringing detection step, the Gaussian parameters were set as $\sigma_{x}=8$ and $\sigma_{y}=8$ in Algorithm 1]. Also, $\delta=0.0001$ and $\ell=5$ so that $11 \times 11$ points 

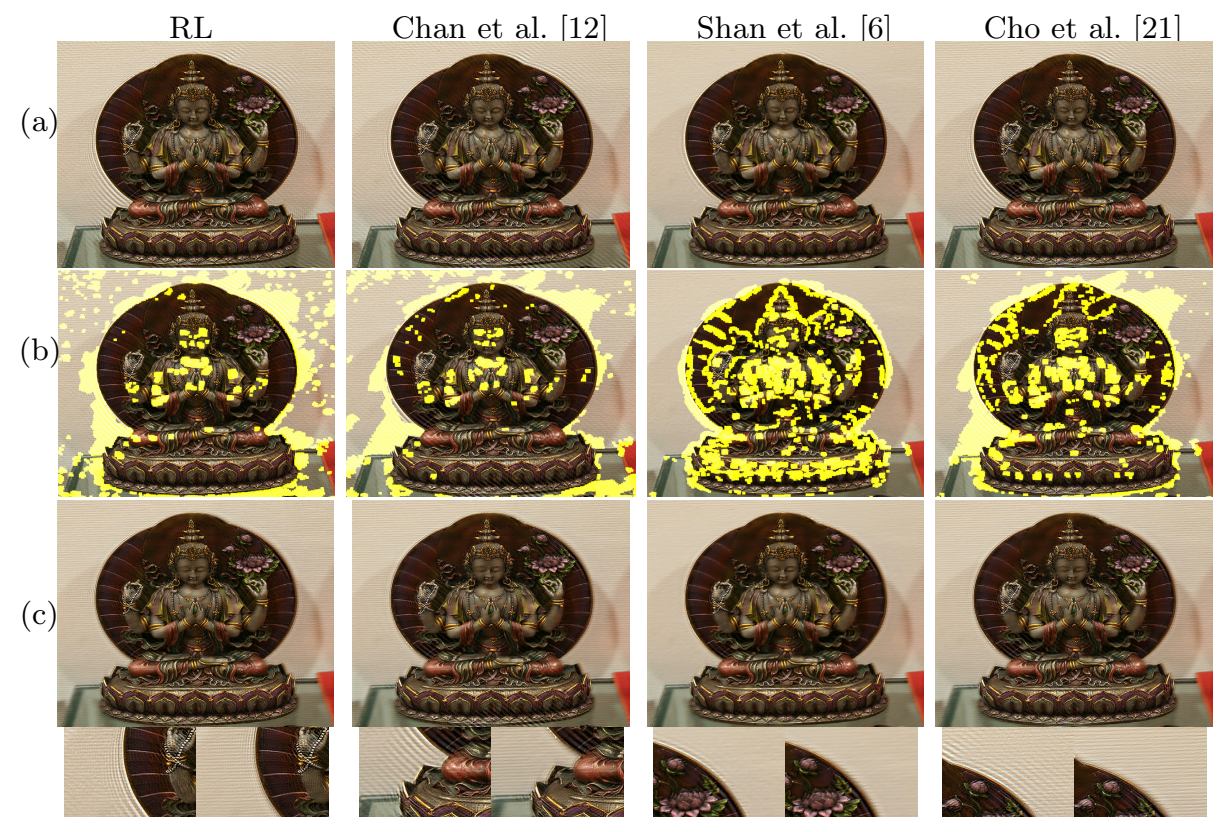

Fig. 6. Deblurring Image 2 using a PSF estimated through blind deconvolution. (The horizontal pattern in the background is part of the original scene and should not be mistaken by ringing patterns.)(a) Deblurring results using different algorithms. (b) Detected ringing regions. (c) Deringing results.

are considered in Eq. (44). Due to the symmetry of the Fourier transform of the PSF, half of the detected minima points can be discarded. This reduces the number of Gabor filters required in the detection process. Ringing artifact detection results are presented in Fig. 5(b), Fig. 6(b), Fig. 7(b). The generated sets of Gabor filters obtained for these examples are shown in Fig. 4(d)-(f). The detected ringing mask for each example is superimposed on the deblurred image with a yellow color. Almost all ringing regions in the deblurred images were detected by the algorithm. The deblurring process is more challenging for the blurry images in Fig. 6 and 7 than for the image presented in Fig. 5, The PSF used in the deblurring process of the image in Fig. 6 was estimated through blind deconvolution. Hence, it may not accurately represent the blur function of the imaging system and can be a different source of ringing artifacts. The same issue may arise from the PSF of Fig. 7 produced using the hybrid-imaging framework. Such PSFs contain sharp discontinuities due to the quantization and interpolation mechanisms employed in the PSF estimation procedure. Moreover, they likely do not represent all of the points of the image [39]. Thus, these PSFs violate the spatial invariance assumption of Eq. (1). Among our test images, the PSF of Image 3 is the extreme case of producing ringing artifacts. Despite such challenges, the ringing artifact detection algorithm detected almost all of the 

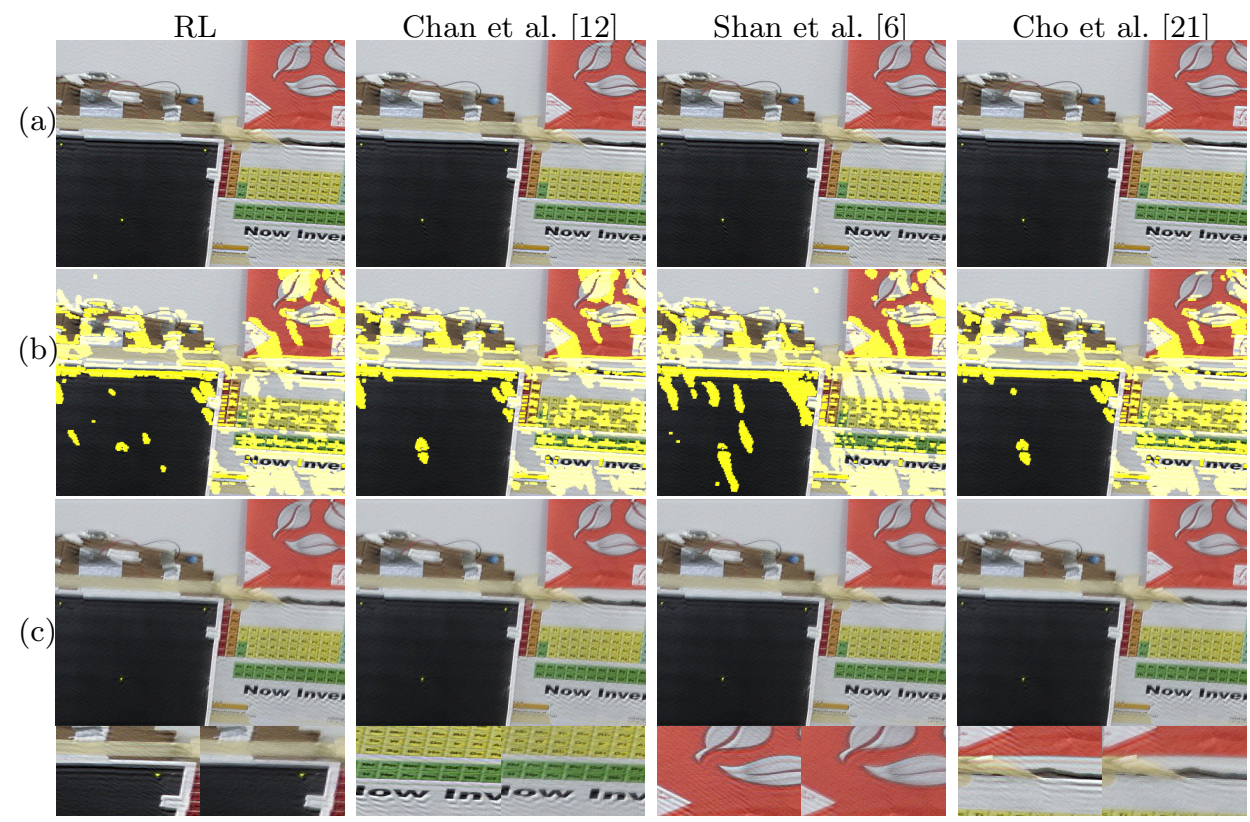

Fig. 7. Deblurring Image 3 using a camera motion-based PSF captured with a hybrid imaging system. (a) Deblurring results using different algorithms. (b) Detected ringing regions. (c) Deringing results

Table 1. Ringing artifact measurement for different algorithms and deringing results

\begin{tabular}{llrrrr}
\hline & Deblurring Algorithms: & RL & Chan et al. [12] & Shan et al. [6] Cho et al. [21] \\
\hline \multirow{2}{*}{ Image 1} & Deblurring & 0.00037 & 0.00210 & 0.00089 & 0.00010 \\
& Deblurring and Deringing & 0.00016 & 0.00022 & 0.00031 & 0.00010 \\
\hline \multirow{2}{*}{ Image 2 2} & Deblurring & 0.02440 & 0.00470 & 0.00112 & 0.00406 \\
& Deblurring and Deringing & 0.00290 & 0.00282 & 0.00091 & 0.00270 \\
\hline \multirow{2}{*}{ Image 3} & Deblurring & 0.00580 & 0.00481 & 0.00673 & 0.00510 \\
& Deblurring and Deringing & 0.00201 & 0.00163 & 0.00210 & 0.00170 \\
\hline
\end{tabular}

ringing regions in the deblurring results of these examples. This is largely due to the fact that artifacts caused by zero-magnitude frequency components of a PSF dominate the ringing patterns present in a deblurred image. Algorithm 2 employs the detected ringing regions and the generated Gabor filters by Algorithm 1 in order to provide a priori knowledge to the regularization scheme to enhance the deblurred images. Algorithm 2 was performed on the deblurring results of the deconvolution schemes. In our simulations, $\beta_{0}=1$ and $\beta_{\max }=64$. Hence, the algorithm runs for 6 iterations. Another important parameter is the weight $\mu$ of the likelihood term in the deringing problem (5). A typical value for $\mu$ is 1000 , so that the process does not remove or over-smooth the essential details of the image. Fig. 5(c), Fig. 6(c) and Fig. 7(c) illustrate the apparent enhancement carried out by the deringing process. 
A quality metric that takes into account the deblurred image ringing artifacts through a non-reference scheme was recently introduced by Lui et al. [28]. In order to provide an objective evaluation, this non-reference quality assessment was used in our experiments. Table 1 summarizes the obtained quality measurements for the deblurred images using different approaches and their post-deconvolution deringing results. A high value obtained for a deblurred image indicates that more perceivable ringing artifacts exist in the image. The values in the table indicate a high performance for the proposed ringing artifact removal algorithm and also the artifact detection algorithm that provides accurate Gabor filters.

Limitations. The method is adapted to the artifacts caused by zeros in the frequency spectrum of the PSF. Hence, it may not detect the ringing patterns generated due to other facts such as non-linearity of a camera response function, non-Gaussian noise, and violating the spatially invariant assumption of the imaging system. Also, the algorithm is customized by considering the local contrast factor to detect the most perceivable ringing artifacts. Therefore, it may miss the ringings that appear in high contrast regions such as the one produced by Chan et al.'s method near the right hand of the statue in Fig 6(b).

\section{Conclusions}

We proposed a new approach to suppress the ringing artifacts produced by image deblurring methods. The artifact removal algorithm is a variational-like regularization scheme that benefits from a novel a priori knowledge in reconstructing an artifact-free image from the deblurred image. The prior is the locations and intensities of the ringing artifacts determined efficiently by a set of Gabor filters. We proposed to generate Gabor filters for each deblurred image with regards to its PSF frequency components. Hence, each produced Gabor filter has specific orientation and frequency details obtained by inspecting the zero-magnitude frequency components of the PSF. Each filter is able to localize a ringing pattern in the deblurred image. We used such filters in the ringing removal process and introduced an independent algorithm to detect ringing artifacts for other purposes such as quality assessment. Our experimental results indicate high performance for both the artifact detection and artifact removal methods.

Although the proposed ringing artifact removal scheme was designed as a post-processing tool to enhance the results of any type of the image deblurring algorithms, the linearity property of Gabor filters makes the introduced prior fairly straight forward to be employed directly in variational-based deconvolution algorithms. The proposed a priori knowledge can be employed along with the total variation norm prior [10], Hyper-Laplacian prior [11], and/or the crosschannel prior 24] in a deconvolution algorithm in order to produce ringing-free deblurred images.

Acknowledgment. This work was supported in part by Mitacs. 


\section{References}

1. Hansen, P.C., Nagy, J.G., O’Leary, D.P.: Deblurring Images: Matrices, Spectra, and Filtering. Society for Industrial and Applied Mathematics (2006)

2. Gonzlez, R.C., Woods, R.E.: Digital Image Processing. Prentice Hall, NJ (2002)

3. Richardson, W.H.: Bayesian-based iterative method of image restoration. J. Opt. Soc. Am. 62(1), 55-59 (1972)

4. Lucy, L.B.: An iterative technique for the rectification of observed distributions. Astronomical Journal 79(6), 745-754 (1974)

5. Mesarovic, V.Z., Galatsanos, N.P., Katsaggelos, A.K.: Regularized constrained total least squares image restoration. IEEE Transactions on Image Processing 4(8), 1096-1108 (1995)

6. Shan, Q., Jia, J., Agarwala, A.: High-quality motion deblurring from a single image. ACM Transactions on Graphics (SIGGRAPGH) 27(3), 73:1-73:10 (2008)

7. Babacan, S.D., Molina, R., Do, M.N., Katsaggelos, A.K.: Bayesian blind deconvolution with general sparse image priors. In: Fitzgibbon, A., Lazebnik, S., Perona, P., Sato, Y., Schmid, C. (eds.) ECCV 2012, Part VI. LNCS, vol. 7577, pp. 341-355. Springer, Heidelberg (2012)

8. Levin, A., Weiss, Y., Durand, F., Freeman, W.T.: Efficient marginal likelihood optimization in blind deconvolution. In: IEEE Conference on Computer Vision and Pattern Recognition (CVPR), pp. 2657-2664 (2011)

9. Rudin, L., Osher, S., Fatemi, E.: Nonlinear total variation based noise removal algorithms. Physica D: Nonlinear Phenomena 60(1), 259-268 (1992)

10. Wang, Y., Yang, J., Yin, W., Zhang, Y.: A new alternating minimization algorithm for total variation image reconstruction. SIAM Journal on Imaging Sciences 1(3), 248-272 (2008)

11. Krishnan, D., Fergus, R.: Fast image deconvolution using hyper-laplacian priors. In: Advances in Neural Information Processing Systems, pp. 1033-1041 (2009)

12. Chan, S.H., Khoshabeh, R., Gibson, K.B., Gill, P.E., Nguyen, T.Q.: An augmented Lagrangian method for total variation video restoration. IEEE Transactions on Image Processing 20(11), 3097-3111 (2011)

13. Chan, T., Golub, G., Mulet, P.: A nonlinear primal-dual method for total variationbased image restoration. SIAM Journal on Scientific Computing 20(6), 1964-1977 (1999)

14. Gregson, J., Heide, F., Hullin, M.B., Rouf, M., Heidrich, W.: Stochastic Deconvolution. In: IEEE Conference on Computer Vision and Pattern Recognition (CVPR), pp. 1043-1050 (June 2013)

15. Yuan, L., Sun, J., Quan, L., Shum, H.Y.: Image deblurring with blurred/noisy image pairs 26(3), 1-10 (2007)

16. Fergus, R., Singh, B., Hertzmann, A., Roweis, S.T., Freeman, W.T.: Removing camera shake from a single photograph 25(3), 787-794 (2006)

17. Levin, A., Weiss, Y., Durand, F., Freeman, W.T.: Understanding and evaluating blind deconvolution algorithms. In: IEEE Conference on Computer Vision and Pattern Recognition (CVPR), pp. 1964-1971 (2009)

18. Levin, A.: Blind motion deblurring using image statistics. In: Advances in Neural Information Processing Systems, pp. 841-848 (2006)

19. Xu, L., Jia, J.: Two-phase kernel estimation for robust motion deblurring. In: Daniilidis, K., Maragos, P., Paragios, N. (eds.) ECCV 2010, Part I. LNCS, vol. 6311, pp. 157-170. Springer, Heidelberg (2010)

20. Yuan, L., Sun, J., Quan, L., Shum, H.Y.: Progressive inter-scale and intra-scale non-blind image deconvolution 27(3), 74:1-74:10 (2008) 
21. Cho, S., Wang, J., Lee, S.: Handling outliers in non-blind image deconvolution. In: IEEE International Conference on Computer Vision (ICCV), pp. 495-502 (2011)

22. Raskar, R., Agrawal, A., Tumblin, J.: Coded exposure photography: motion deblurring using fluttered shutter. ACM Transactions on Graphics (SIGGRAPH) 25, 795-804 (2006)

23. Levin, A., Fergus, R., Durand, F., Freeman, W.T.: Image and depth from a conventional camera with a coded aperture. ACM Transactions on Graphics (SIGGRAPH) 26(3), 70 (2007)

24. Heide, F., Rouf, M., Hullin, M.B., Labitzke, B., Heidrich, W., Kolb, A.: Highquality computational imaging through simple lenses. ACM Transactions on Graphics (SIGGRAPH) (2013)

25. Liu, H., Klomp, N., Heynderickx, I.: A perceptually relevant approach to ringing region detection. IEEE Transactions on Image Peocessing 19(6), 1414-1426 (2010)

26. Liu, H., Klomp, N., Heynderickx, I.: A no-reference metric for perceived ringing artifacts in images. IEEE Transactions on Circuits and Systems for Video Technology 20(4), 529-539 (2010)

27. Zuo, B.X., Ming, D.L., Tian, J.W.: Perceptual ringing metric to evaluate the quality of images restored using blind deconvolution algorithms. Optical Engineering 48(3), 037004-037004 (2009)

28. Liu, Y., Wang, J., Cho, S., Finkelstein, A., Rusinkiewicz, S.: A no-reference metric for evaluating the quality of motion deblurring. ACM Transactions on Graphics (SIGGRAPH Asia) (2013)

29. Jain, A.K., Farrokhnia, F.: Unsupervised texture segmentation using gabor filters. In: IEEE International Conference on Systems, Man and Cybernetics, pp. 14-19 (1990)

30. Peli, E.: Contrast sensitivity function and image discrimination. Journal of Optical Society of America 18(2), 283-293 (2001)

31. Tang, J., Kim, J., Peli, E.: Image enhancement in the JPEG domain for people with vision impairment. IEEE Transactions on Biomedical Engineering 51(11), 2013-2023 (2004)

32. Reinhard, E., Shirley, P., Ashikhmin, M., Troscianko, T.: Second order image statistics in computer graphics. In: Symposium on Applied Perception in Graphics and Visualization, pp. 99-106 (2004)

33. Tikhonov, A., Arsenin, V.: Solutions of Ill-posed Problems. Winston and Sons, Washington (1977)

34. Goldstein, T., Osher, S.: The split Bsregman method for L1-regularized problems. SIAM Journal on Imaging Sciences 2(2), 323-343 (2009)

35. Geman, D., Reynolds, G.: Constrained restoration and the recovery of discontinuities. IEEE Transactions on Pattern Analysis and Machine Intelligence 14(3), 367-383 (1992)

36. Geman, D., Yang, C.: Nonlinear image recovery with half-quadratic regularization. IEEE Transactions on Image Processing 4(7), 932-946 (1995)

37. Yang, J., Zhang, Y., Yin, W.: An efficient TVL1 algorithm for deblurring multichannel images corrupted by impulsive noise. SIAM Journal on Scientific Computing 31(4), 2842-2865 (2009)

38. Ben-Ezra, M., Nayar, S.: Motion-based motion deblurring. IEEE Transactions on Pattern Analysis and Machine Intelligence 26(6), 689-698 (2004)

39. Tai, Y.W., Du, H., Brown, M.S., Lin, S.: Image/video deblurring using a hybrid camera. In: IEEE Conference on Computer Vision and Pattern Recognition (CVPR), pp. 1-8 (2008) 